\title{
Study on Hough Transform in XXX Radar Signal Detection
}

\author{
Ding Qiuqi, Jiang Zhiyu
}

China Satellite Maritime Tracking and Control Department, Jiangyin, 214431, China

Keywords: Optimal allocation; signal detection

\begin{abstract}
In this paper, the key issues of the classical Hough transform XXX radar signal detection were studied, focusing on the parameter space is divided phenomenon, given to find the optimal partitioning method for XXX and radar to find the best way to divide and give a rational method of extracting the peak. So as to achieve optimal Hough accumulation assay provides favorable conditions.
\end{abstract}

\section{Introduction}

Reasonable threshold setting can further improve the processing speed. Dividing the parameter space not only affects the computation algorithm, but also can affect the detection performance, this is the biggest factor Hough transform effects for different radars have different classification methods. In this paper, the key issues of the classical Hough transform XXX radar signal detection were studied, focusing on the parameter space is divided phenomenon, given to find the optimal partitioning method for XXX and radar to find the best way to divide and give a rational method of extracting the peak. So as to achieve optimal Hough accumulation assay provides favorable conditions.

\section{Threshold Selection}

Hough accumulation was detected by dual threshold detection; different threshold will affect the speed of the algorithm and detection performance. If the first threshold is set too high, although the exclusion of a lot of noise, reducing the amount of subsequent calculations, but too much will cause the target signal is lost, the second threshold is necessary to set a lower some, this may result in the accumulation of too low peak missed ; if the first threshold is set too low, while ensuring the target signal is not lost, but will introduce excessive noise caused by the subsequent increase in the amount of calculation, and to the second threshold is set too high in order to reduce the impact of noise on accumulation. FIG.1 (a) and 1 (b), the input SNR 6dB respectively, the first threshold is set to a first threshold processing results to $10^{-3}$ and $10^{-2}$. See, (a) in FIG.1 the target signal loss than in FIG.1 (b) in much lower if the SNR, this loss may be more, even if the second threshold is reduced, since the decrease in signal accumulation such that the detection performance. Literature has made the corresponding research and simulation of a first threshold, the second threshold and detection probability relations, pointed out that in Rayleigh and sea clutter, when the first threshold is 10-1 when the maximum detection probability, but in order to reduce the subsequent computational algorithm, usually the first threshold is $10-2$, then the detection performance degradation is small and a large amount of calculation is not introduced.

It should be noted that the above we discussed threshold size are used in the form of the probability of false alarm rather than $\mathrm{dB}$ value, which is due to discuss the form of signal to noise ratio greater arbitrariness, inconvenience compare with other detectors. Rayleigh clutter distribution discussed in this paper, the probability of false alarm $10^{-2}$ corresponding to the signal to noise ratio is about $6.6 \mathrm{~dB}$.

In addition to the selection threshold method, you can also use an adaptive threshold method. First calculate the average background noise $u_{1}$, Groups threshold is set to $\eta=n_{1} u_{1}$, where $n_{1}$ is a coefficient larger than 1 , such as 2.5 can be set; and then calculating the mean accumulation $\rho-\theta$ plane $u_{2}$, the second threshold is set to $\zeta=n_{2} u_{2}, n_{2}$ is still a coefficient larger than 1 , and 
there is $n_{1}<n_{2}$, as may be set at 5 . Select different thresholds would be false alarm probability and detection probability of an impact.

In practice, the use of adaptive threshold method more flexible, but requires a lot of experiments to select the appropriate coefficients. In this paper, for simplicity and more focused, we select the probability of false alarm as a threshold criterion. On the second selected threshold, we give the best division in the parameter space.

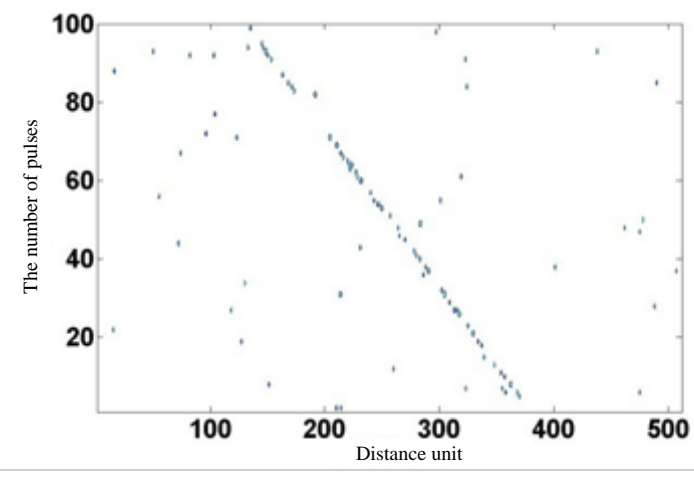

(A). The probability of false alarm of $10^{-3}$ when processing results is $10^{-2}$ when processing results

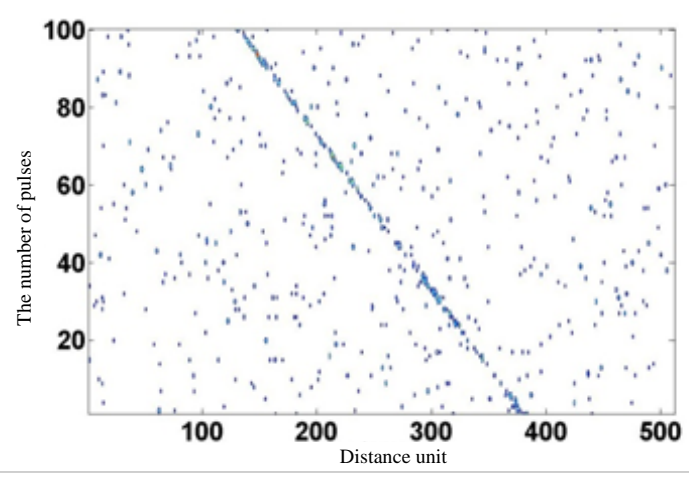

(B). False alarm probability

Figure 1. The first threshold under different processing result data space

\section{Optimal dividing the parameter space}

Different in practice, since the data is discrete and therefore the need for discrete parameter space is divided, different radar parameters and accumulated number of pulses need data space, and different data space is divided to correspond to different parameter space. Obviously, dividing the parameter space detection performance is directly related to the algorithm: too sparse division can result in excess noise aliased parameters accumulation unit, and thus the need to improve a second threshold; and the division is too fine, not only increases the amount of computation, but also leads to the point of falling into the same line of different parameters accumulation unit so that echo energy in the same straight line can not be effectively accumulated. Since the peak position and influence of noise, echo happen "dithering", making the mapped curve may not intersect at one point, but distributed in a small area around the theoretical point. Therefore we need to find an optimal parameter classification method.

XXX parameters for radar, this paper uses data space $100 \times 512$, that is 100 pulse-echo wave gate 512 sampling points. The reason is that, first, the non-coherent integration of theoretical SNR gain $\mathrm{dB}$, when the accumulated number of 100 SNR gain of $10 \mathrm{~dB}$, although the increase in the number of accumulated pulses can improve the signal to noise ratio, but will greatly increase the amount of calculation, and noncoherent SNR gain has not greatly improved, for example, even when the accumulated pulses to 1000 , but also the SNR $15 \mathrm{~dB}$, and in order not to run out of the target wave gate wave gate width must also be increased, resulting in the calculation amount is greatly increased. Secondly, when the signal to noise ratio is less than $0 \mathrm{~dB}$, noncoherent poor performance, so in order to improve the SNR gain by simply increasing the number of accumulated pulses is obviously not "cost effective." Again, for the XXX radar, the sampling rate is $20 \mathrm{MHz}$, wave gate 512 sampling points, the accumulation time can be guaranteed not to leave the target gates. For these reasons, we will deal with the data space size as $100 \times 512$.

Before the introduction of the parameter space optimal partitioning method, to illustrate the concept of up Hough space. Up Hough space (accessible Hough space) indicates that the data space can be mapped to the set of parameter space unit, in short, all the parameter space may not be set to 0 . unit. The reason why the concept of up Hough space, because in the parameter space of some of the units in the corresponding linear data space could not exist in the parameter space of these points are always 0 , so that during the time of theoretical analysis to exclude these points, and different parameters unit may accumulate points are different. Obtaining specific method: All units 
assigned to the data space is 1, then the data space for all units Hough transform parameter space so that all cells greater than 0 is set up Hough space, as shown in FIG. The maximum number of data space unit value of up to Hough space is a unit that can be mapped.

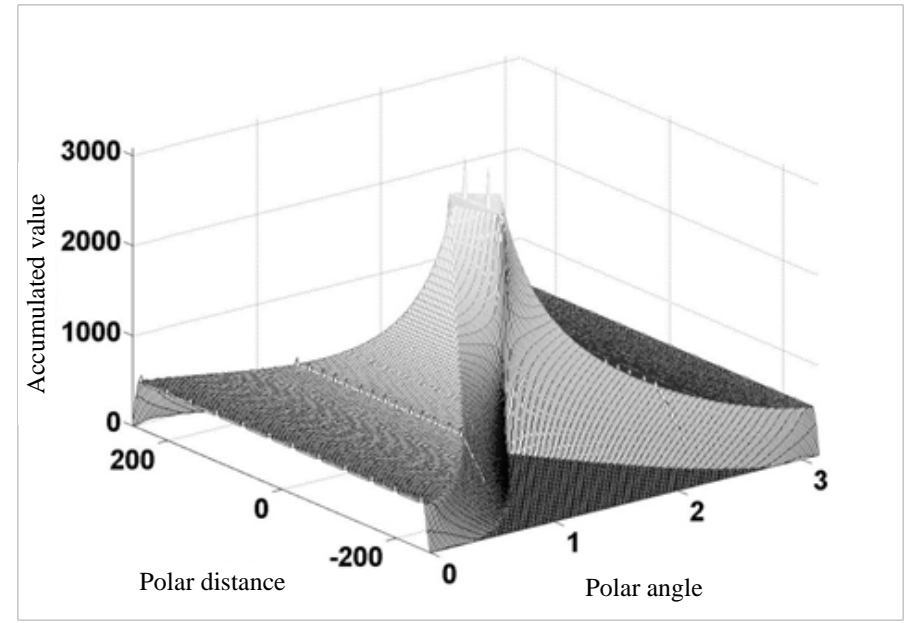

Figure 2. The schematic diagram up Hough space

Now consider a single Hough false alarm probability and detection probability of the cell, non-fluctuating targets in noise power exponential distribution, the probability of false alarm unit single Hough is:

$$
P_{\mathrm{F}}=1-\left(1-p_{\mathrm{F}}\right)^{N}-\sum_{m=1}^{m^{*}-1}\left(\begin{array}{l}
N \\
m
\end{array}\right)\left(1-p_{\mathrm{F}}\right)^{N-m} p_{\mathrm{F}}^{m} \times\left[1-e^{-(\xi-m \eta)} \sum_{\ell=0}^{m-1} \frac{(\xi-m \eta)^{\ell}}{\ell !}\right]
$$

Formula $\mathrm{N}$ is the maximum number of data can be mapped to spatial units on the parameter unit, whose value is up to Hough parameter space corresponding to the unit value. To reflect the probability of false alarm throughout the Hough space, the relevant literature suggests that the average probability of false alarm of the concept and its formula was correct to give:

$$
P_{\text {Ftotal }}=\sum_{1}^{N_{\text {max }}} w_{N}^{N} P_{\mathrm{F}} / \sum_{N=1}^{N_{\max }} w_{N}
$$

Hough single unit detection probability is:

$$
P_{\mathrm{D}}=1-\left(1-p_{\mathrm{D}}\right)^{N}-\sum_{m=1}^{m^{*}-1}\left(\begin{array}{l}
N \\
m
\end{array}\right)\left(1-p_{\mathrm{D}}\right)^{N-m} \times\left[p_{\mathrm{D}}^{m}-e^{-\xi-m s} \sum_{k=0}^{\infty} c_{k} \sum_{\ell=0}^{m+k-1} \frac{(\xi-m \eta)^{\ell}}{\ell !}\right]
$$

The method of calculating the coefficient $c_{k}$ is as follows:

$$
\begin{aligned}
& c_{0}=a_{0}^{m} \\
& c_{k}=\frac{1}{k a_{0}} \sum_{\ell=1}^{k}(\ell m-k+\ell) a_{\ell} c_{k-\ell} \\
& a_{j}=(\sqrt{S / \eta})^{j} I_{j}(2 \sqrt{S \eta})
\end{aligned}
$$

For specific data space, to find the optimal parameter space is divided into the basic idea: the use of different methods of parameter space is divided Hough transform, to keep all the B constant, changing the input signal to noise ratio to obtain the same $\mathrm{C}$, so that we can get through the calculation a matrix $\mathrm{D}$, where $\mathrm{E}$ is the corresponding parameter space into $\mathrm{F}$ is the optimal division. We are looking for the optimal parameter space division during the second threshold $\mathrm{G}$ along with it OK. To this end we have given a hypothetical target speed of $5.5 \mathrm{~km} / \mathrm{s}$, so that $\mathrm{HH}=9$. After calculation, we obtain the optimal parameters of the data division in the space of $100 \times 512: \mathrm{J}=0$, the second threshold is $\mathrm{K}=4$.

For specific data space, to find the optimal parameter space is divided into the basic idea: the use of different methods of parameter space is divided Hough transform, to keep all the $P_{\text {Ftotal }}$ constant, 
changing the input signal to noise ratio to obtain the same $P_{D}$, so that we can get through the calculation a matrix $\left(\Delta \rho_{i}, \Delta \theta_{i}, S_{i}\right)$, where $\min \left(\left\{S_{i}\right\}\right)$ is the corresponding parameter space into $\left(\Delta \rho_{i}, \Delta \theta_{i}\right)$ is the optimal division. We are looking for the optimal parameter space division during the second threshold $\zeta$ along with it OK. To this end we have given a hypothetical target speed of $5.5 \mathrm{~km} / \mathrm{s}$, so that $P_{\mathrm{f}}=10^{-2}, P_{\text {Ftotal }}=10^{-6}, P_{\mathrm{D}}=0.9$. After calculation, we obtain the optimal parameters of the data division in the space of $100 \times 512: \Delta \rho=5, \Delta \theta=0.75^{\circ}$, the second threshold is $\zeta=23 \mathrm{~dB}$.

For Hough two accumulated value, the literature also derived probability of detection and false alarm probability formula for the optimal parameter space division provides a theoretical basis, no further explanation. For convenience, Hough two are accumulated value detecting method using the above division, we obtain by calculation a second threshold when the accumulated value of the two is equal to 48 .

\section{Summary}

This chapter of the non-coherent integration algorithm based on the Hough transform were studied. Research on the key issues Hough Transform XXX radar signal detection, and focus on the optimal parameter space partitioning problem and address this issue gives the optimal parameter space division method. The situation of uniform motion and constant acceleration motion simulation carried out, the results proved the uniform motion detection performance at best, uniformly accelerated motion detection performance is slightly inferior, but radar can improve the ability to detect a certain extent.

\section{Reference:}

[1] Xiang-Gen Xia, Discrete Chirp-Fourier Transform and its Application to Chirp Rate Estimation [J]. IEEE Trans. On Signal Processing, 2010, 48(11): 3122-3133

[2] J.Illingworth, J.Kittler. A Survey of the Hough Transform [J]. COMPUTER VISION, GRAPHICS, AND IMAGE PROCESSING, 2008, 44: 87-116

[3] John Immerker. Some remarks on the straight line Hough transform. Pattern Recognition Letters, 2008, 19: 1133-1135

[4] Duda, R. O., Hart, et al. Use of the Hough transformation to detect lines and curves in pictures. Communication of the ACM, 2012, 15(1): 11-15

[5] Carlson B.D., E.D. Evans, S.L. Wilson. Search Radar Detection and Track with the Hough Transform, Part III: Detection Performance with Binary Integration [J] IEEE Trans. On Aerospace and Electronic systems, 2013, 30(1): 116-124

[6] Moshe Elazar. Search Radar Tranck-before-Detect Using the Hough Transform. ADA 295245, 2013 